\title{
Correction: In silico design of novel gold-phosphate containing compounds as selective inhibitors of cathepsin B in neuroinflammation
}

\author{
Alexsey V. Raevsky ${ }^{1}$, Mohsen Sharifi' ${ }^{2,3}$, Vasily Pinchuk ${ }^{4}$, Andis Klegeris ${ }^{5}$ \\ 'Laboratory of Structural Biology, Institute of Food Biotechnology and Genomics, National Academy of Sciences of Ukraine, Kyiv 04123, \\ Ukraine. \\ ${ }^{2}$ Medway School of Pharmacy, Universities of Kent and Greenwich, Kent ME4 4TB, UK. \\ ${ }^{3}$ Present address: Division of Systems Biology, National Center for Toxicological Research, US Food and Drug Administration, Jefferson, \\ AR 72079, USA. \\ ${ }^{4}$ Life Chemicals Ltd., Niagara-on-the-Lake, ON LOS 1J0, Canada. \\ ${ }^{5}$ Department of Biology, University of British Columbia, Okanagan Campus, Kelowna, BC V1V 1V7, Canada. \\ Correspondence to: Dr. Alexsey V. Raevsky, Laboratory of Structural Biology, Institute of Food Biotechnology and Genomics, National \\ Academy of Sciences of Ukraine, Kyiv 04123, Ukraine. E-mail: o.v.raievskyi@imbg.org.ua; Dr. Andis Klegeris, Department of Biology, \\ University of British Columbia, Okanagan Campus, Kelowna, BC V1V 1V7, Canada. E-mail: andis.klegeris@ubc.ca
}

How to cite this article: Raevsky AV, Sharifi M, Pinchuk V, Klegeris A. In silico design of novel gold-phosphate containing compounds as selective inhibitors of cathepsin B in neuroinflammation. Neuroimmuno/ Neuroinflammation 2018;5:44.

http://dx.doi.org/10.20517/2347-8659.2018.61

Revised: 28 Sep 2018 Accepted: 30 Sep 2018 Published: 22 Oct 2018

The original article was published on 27 Aug 2018.

Dr. Mohsen Sharifi declares the view presented in the article are those of the authors and do not reflect those of the US Food and Drug Administration. No official endorsement is intended nor should be inferred. 